\title{
AVERAGING TECHNIQUES WITHOUT REQUIRING A FAST TIME-VARYING DIFFERENTIAL EQUATION *
}

\author{
Joan Peuteman ${ }^{a}$, Dirk Aeyels ${ }^{b}$ \\ ${ }^{a}$ Katholieke Hogeschool Brugge Oostende, Departement IWET, lab ECOREA, Zeedijk 101, Oostende B-8400, Belgium \\ b SYSTeMS Research Group, Universiteit Gent, Technologiepark 914, B-9042 Zwijnaarde, Belgium
}

\begin{abstract}
An averaging result is presented for local uniform asymptotic stability of nonlinear differential equations without requiring a fast time-varying vectorfield. The nonlinearity plays a crucial role: close to the origin, the trajectories vary slowly compared to the time dependence of the vectorfield. The result generalises averaging results which prove stability properties for systems having a homogeneous vectorfield with positive order. The result is illustrated with several examples.
\end{abstract}

Key words: Asymptotic stability; time-varying; averaging; Liapunov

\section{Introduction}

It is well known that a solution of a time-varying system $\dot{x}(t)=\varepsilon f(x(t), t)$ may be approximated by the solution of the averaged system $\dot{x}(t)=\varepsilon \bar{f}(x(t))$ on a large time-scale for $\varepsilon$ sufficiently small, $[5,10,3]$. The averaging technique also provides a tool to investigate exponential stability of an equilibrium of $\dot{x}(t)=\varepsilon f(x(t), t)$ for $\varepsilon$ sufficiently small i.e. exponential stability of the averaged system $\dot{x}(t)=\varepsilon \bar{f}(x(t))$ implies exponential stability of the original time-varying system $[1,5]$. In other words, for $\varepsilon$ sufficiently small, exponential stability of the equilibrium point $x=0$ of $\dot{x}(t)=\bar{f}(x(t))$ implies exponential stability of $x=0$ of the original fast timevarying system $\dot{x}(t)=f(x(t), t / \epsilon)$. Averaging results are also available for nonsmooth systems when using dither, where solutions of the averaged system (with respect to dither frequency) approximate solutions of the original system [4].

The averaging concept is useful not only in relation to exponential stability, but also when investigating practical stability properties [9] and uniform asymptotic stability properties [8]. In [6,7] the averaging technique is applied to homogeneous systems of order $\tau=0$. Homo-

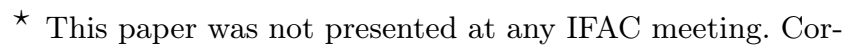
responding author is D. Aeyels. Tel. +32 9264 5656, Fax. $+3292645840$
}

Email addresses: joan.peuteman@khbo.be (Joan Peuteman), dirk.aeyels@ugent.be (Dirk Aeyels). geneous systems with order $\tau>0$ can also be dealt with: in [8], it is shown that asymptotic stability of the averaged homogeneous system implies local uniform asymptotic stability of the original time-varying homogeneous system without requiring that the original system is fast time-varying.

In this paper, the averaging results discussed in [8] are generalised. Under extra conditions on the differential equation, but without requiring homogeneity of the system, we will show that local asymptotic stability of the averaged system implies local uniform asymptotic stability of the original time varying system. This original time-varying system need not be fast time-varying. Appropriate conditions on the vector field in terms of class $K$ functions imply the local uniform asymptotic stability property without requiring a fast time-varying vectorfield or homogeneity with order $\tau>0$. What is needed is that, sufficiently close to the equilibrium point $x=0$, the trajectories are slowly varying compared to the time dependence of the vectorfield. This is usually accomplished through the introduction of a parameter $\epsilon$ as indicated above; our main contribution is that in the averaging approach the role played by $\epsilon$ may be assumed by the vector field itself.

A number of different examples are included to illustrate our main result and in particular to indicate how it is a generalisation of the homogeneous result formulated in [8]. 


\section{The main averaging result}

Consider

$$
\dot{x}(t)=f(x(t), t)
$$

with $f: W \times \mathbf{R}^{+} \rightarrow \mathbf{R}^{n}, W$ is an open and convex set, $W \subset \mathbf{R}^{n}$. Let $0 \in W$ and $f(0, t)=0$ for all $t \in \mathbf{R}^{+}$. Furthermore, we assume that conditions are imposed on (1) such that existence and uniqueness of its solutions are secured (existence and uniqueness is a standard assumption for all the systems considered in this paper). The system (1) is time-periodic i.e. there exists a $T>0$ such that for all $x \in W$ and for all $t \in \mathbf{R}^{+}$,

$$
f(x, t)=f(x, t+T) .
$$

For all $x \in W$, define the averaged system as

$$
\dot{x}(t)=\bar{f}(x(t))
$$

where for all $x \in W$,

$$
\bar{f}(x)=\frac{1}{T} \int_{0}^{T} f(x, t) d t .
$$

We recall when a continuous function is said to belong to class $K$ or to class $K L$ :

The continuous function $\alpha:[0, a) \rightarrow \mathbf{R}^{+}$(for some $a>$ $0)$ is a class $K$ function if it is strictly increasing and $\alpha(0)=0$.

The continuous function $\beta:[0, a) \times \mathbf{R}^{+} \rightarrow \mathbf{R}^{+}$(for some $a>0)$ is a class $K L$ function if:

(1) for each fixed $s, \beta(r, s)$ is a class $K$ function in $r$

(2) for each fixed $r$, the function $\beta(r, s)$ is decreasing in $s$ and $\beta(r, s) \rightarrow 0$ as $s \rightarrow+\infty$.

The equilibrium $x=0$ of (1) is locally uniformly asymptotically stable if there exists a class $K L$ function $\beta$ and a positive constant $c$ such that $\forall t_{0} \geq 0$

$$
\left\|x\left(t_{0}\right)\right\|<c \Rightarrow\|x(t)\| \leq \beta\left(x\left(t_{0}\right), t-t_{0}\right), \forall t \geq t_{0} .
$$

When for equation (3) there exists $t_{0} \geq 0$ for which (5) is true, then (5) is true $\forall t_{0} \geq 0$ : one says that the zero equilibrium of (3) is locally asymptotically stable.

For the $\epsilon-\delta$ definitions of local (uniform) asymptotic stability, the reader is referred to [5].

From Liapunov theory we know that the equilibrium point $x=0$ of (3) is locally asymptotically stable when there exists an open neighborhood $U \subset W$ of 0 and there exists a Liapunov function $V: U \rightarrow \mathbf{R}$ such that for all $x \in U$ :

$$
\alpha_{1}(\|x\|) \leq V(x) \leq \alpha_{2}(\|x\|),
$$

$$
\frac{\partial V}{\partial x}(x) \bar{f}(x) \leq-\alpha_{3}(\|x\|)
$$

Here, $\alpha_{1}, \alpha_{2}, \alpha_{3}: \mathbf{R}^{+} \rightarrow \mathbf{R}^{+}$are class $K$ functions.

Main theorem: Assume that the following conditions are satisfied:

- the equilibrium point $x=0$ of the averaged system (3) is locally asymptotically stable, equivalently: (6) and (7) are satisfied,

- there exists a class $K$ function $\alpha_{4}: \mathbf{R}^{+} \rightarrow \mathbf{R}^{+}$such that for all $x \in U$

$$
\left\|\frac{\partial V}{\partial x}(x)\right\| \leq \alpha_{4}(\|x\|),
$$

- $f$ is continuously differentiable with respect to $x$ on $W$ for all $t \in \mathbf{R}^{+}$; furthermore: for all $x \in W$ and for all $t \in \mathbf{R}^{+}$

$$
\left\|\frac{\partial f}{\partial x}(x, t)\right\| \leq \alpha_{5}(\|x\|),
$$

where, $\alpha_{5}: \mathbf{R}^{+} \rightarrow \mathbf{R}^{+}$is a class $K$ function with the additional property that sufficiently close to the origin $\alpha_{5}\left(\|x\|\left(1+2 T \alpha_{5}(\|x\|)\right)\right) \leq 2 \alpha_{5}(\|x\|)$,

- the function

$$
\alpha_{6}(\|x\|) \triangleq \frac{\alpha_{4}(\|x\|) \alpha_{5}^{2}(\|x\|)\|x\|}{\alpha_{3}(\|x\|)}
$$

is a class $K$ function $\left(\alpha_{6}(0) \triangleq 0\right)$.

Then the equilibrium point $x=0$ of the original system (1) is locally uniformly asymptotically stable.

The three remarks that follow aim to assess the meaning and significance of condition (9) and (10), and discuss why conditions (9) and (10) are not compatible with linear systems.

Remark 1: In order to conclude that local asymptotic stability of the equilibrium point of the averaged system (3) implies local uniform asymptotic stability of the equilibrium point of the original system (1) without requiring a fast time-varying vectorfield, conditions (9) and (10) are crucial. Condition (9) implies that sufficiently close to the equilibrium point, the trajectories 
vary slowly in time, compared to the time-dependence of the vectorfield. Condition (9) generalises the homogeneous conditions proposed in [8] where a positive order $\tau>0$ is required. Condition (10) is more technical and plays a crucial role in part VI of the proof.

Remark 2: We discuss the feasibility of the technical condition that sufficiently close to the origin $\alpha_{5}\left(\|x\|\left(1+2 T \alpha_{5}(\|x\|)\right)\right) \leq 2 \alpha_{5}(\|x\|)$. In case $\alpha_{5}$ is continuously differentiable, it is possible to prove this technical condition using the mean value theorem. By continuous differentiability, $\alpha_{5}^{\prime}$ is bounded on every arbitrary compact set $[0, r]$. The mean value theorem implies the existence of a $z \in\left(\|x\|,\|x\|\left(1+2 T \alpha_{5}(\|x\|)\right)\right)$ such that

$$
\begin{aligned}
& \alpha_{5}\left(\|x\|\left(1+2 T \alpha_{5}(\|x\|)\right)\right)= \\
& \alpha_{5}(\|x\|)+2 T \alpha_{5}(\|x\|)\|x\| \alpha_{5}^{\prime}(z) .
\end{aligned}
$$

Starting with a fixed $r$, suppose $M$ is an upper bound for $\left|\alpha_{5}^{\prime}\right|$ on $[0, r]$. Taking $\|x\|$ sufficiently small such that $\|x\|\left(1+2 T \alpha_{5}(\|x\|)\right)<r$, one obtains that $\left|\alpha_{5}^{\prime}(z)\right| \leq$ $M$. With the additional condition that $\|x\| \leq 1 / 2 T M$ such that $2 T \alpha_{5}(\|x\|)\|x\|\left|\alpha_{5}^{\prime}(z)\right| \leq \alpha_{5}(\|x\|)$, the technical condition is satisfied.

Example 1 (see (82)) and Example 2 (see (94)) also illustrate the feasibility of this technical condition.

Remark 3: For a linear system and using a quadratic Liapunov function $V, \alpha_{3}$ is a quadratic function and $\alpha_{4}$ is a linear function. Condition (9) is not satisfied since $\partial f / \partial x$ is nonzero at the origin. By replacing $\alpha_{5}$ in (9) by a constant bound, also expression (10) does not provide a class $\mathrm{K}$ function (one obtains a constant). The main theorem does not prove stability properties: linear systems require fast time-varying vectorfields in order to obtain averaging results $[1,5]$.

\section{Outline of the proof:}

First, an appropriate change of variables (21) is defined. This leads to the system (26) in $y$ which is equivalent with the original system (1) in $x$. Since (26) may be seen as a perturbation of the averaged system (3), the Liapunov function $V$ is invoked to prove local uniform asymptotic stability of the equilibrium point of (26). Formulating this stability property in terms of class KL functions, and since the change of variables (21) defines a one-to-one relationship, also the evolution of the trajectories of (1) are bounded by class KL functions. This implies local uniform asymptotic stability of the equilibrium point of (1).

\section{Proof:}

\section{I: Preliminary definitions and some useful bounds}

For all $y \in W$ and for all $t \in \mathbf{R}^{+}$define

$$
h(y, t) \triangleq f(y, t)-\bar{f}(y)
$$

and

$$
u(y, t) \triangleq \int_{0}^{t} h(y, \tau) d \tau=\int_{0}^{t}(f(y, \tau)-\bar{f}(y)) d \tau
$$

Since $h(y, t)$ is $T$-periodic in $t$ and has zero mean, $u(y, t)$ is also $T$-periodic. Indeed, for all $t \in \mathbf{R}^{+}$there exists a $n \in \mathbf{N}$ such that $0 \leq t-n T<T$ and

$$
\begin{array}{r}
u(y, t)=\int_{0}^{n T} h(y, \tau) d \tau+\int_{n T}^{t} h(y, \tau) d \tau= \\
\int_{n T}^{t} h(y, \tau) d \tau .
\end{array}
$$

Moreover, $u(y, t)$ is continuous in $t$.

We first establish some inequalities to be used later on. Expression (9) and (Lemma 3.1 on pp. 89-90 of [5]) imply that for all $y_{1}, y_{2} \in W$ (W is convex) and for all $t \in \mathbf{R}^{+}$

$$
\begin{aligned}
& \left\|f\left(y_{1}, t\right)-f\left(y_{2}, t\right)\right\| \leq \\
& \max \left(\alpha_{5}\left(\left\|y_{1}\right\|\right), \alpha_{5}\left(\left\|y_{2}\right\|\right)\right)\left\|y_{1}-y_{2}\right\| .
\end{aligned}
$$

Indeed, the time-periodic $\frac{\partial f}{\partial x}$ exists and is continuous on the line segment $L_{y_{1} y_{2}}$ defined as $\left\{\alpha y_{1}+(1-\alpha) y_{2}\right.$ : $\alpha \in[0,1]\}$. For all $y \in L_{y_{1} y_{2}} \subset W$, there exists an $\alpha \in[0,1]$ such that $\|y\| \leq \alpha\left\|y_{1}\right\|+(1-$ $\alpha)\left\|y_{2}\right\| \leq \max \left(\left\|y_{1}\right\|,\left\|y_{2}\right\|\right)$. From (9), this implies that $\left.\left\|\frac{\partial f}{\partial x}(x, t)\right\|\right|_{x=y} \leq \max \left(\alpha_{5}\left(\left\|y_{1}\right\|\right), \alpha_{5}\left(\left\|y_{2}\right\|\right)\right)$ for all $y \in L_{y_{1} y_{2}}$, implying (15).

From (15), one obtains that $\|f(y, t)\| \leq \alpha_{5}(\|y\|)\|y\|$ for all $y \in W$ and for all $t \in \mathbf{R}^{+}$, implying that

$$
\begin{aligned}
\|\bar{f}(y)\| & \leq \frac{1}{T} \int_{0}^{T}\|f(y, \tau)\| d \tau \leq \\
\frac{1}{T} \int_{0}^{T} \alpha_{5}(\|y\|)\|y\| d \tau & \leq \alpha_{5}(\|y\|)\|y\| .
\end{aligned}
$$

From (4) and (9), one obtains that for all $y \in W$

$$
\begin{aligned}
\left\|\frac{\partial \bar{f}}{\partial y}(y)\right\| \leq \frac{1}{T} \int_{0}^{T}\left\|\frac{\partial f}{\partial y}(y, t)\right\| d t \leq & \\
\frac{1}{T} \int_{0}^{T} \alpha_{5}(\|y\|) d t & =\alpha_{5}(\|y\|) .
\end{aligned}
$$

Let $n \in \mathbf{N}$ be such that $0 \leq t-n T<T$. From (13), (14), (15) and (16), one obtains that for all $y \in W$ and 
$\|u(y, t)\| \leq \int_{n T}^{t}(\|f(y, \tau)\|+\|\bar{f}(y)\|) d \tau \leq 2 T \alpha_{5}(\|y\|)\|y\|$

From (14) one obtains for all $y \in W$ and for all $t \in \mathbf{R}^{+}$ that

$$
\frac{\partial u}{\partial y}(y, t)=\int_{n T}^{t}\left(\frac{\partial f}{\partial y}(y, \tau)-\frac{\partial \bar{f}}{\partial y}(y)\right) d \tau
$$

and therefore, invoking (9) and (17),

$$
\left\|\frac{\partial u}{\partial y}(y, t)\right\| \leq \int_{n T}^{t}\left(\left\|\frac{\partial f}{\partial y}(y, \tau)\right\|+\underset{\left.2 T \alpha_{5}(\|y\|) \cdot \quad\left\|\frac{\partial \bar{f}}{\partial y}(y)\right\|\right) d \tau \leq}{(20)}\right.
$$

\section{II: System (1) with a change of variables}

With $u(y, t)$ defined in (13), consider the change of variables

$$
x=y+u(y, t) .
$$

Using this change of variables, the system (1) in $x$ will be equivalent with (26) in $y$. Since (26) may be seen as a perturbation of the averaged system (3), the Liapunov function $V$ may be used to prove local uniform asymptotic stability of the equilibrium point of (26).

Consider the continuous strictly increasing function $\alpha_{7}$ : $\mathbf{R}^{+} \rightarrow \mathbf{R}^{+}$with $\alpha_{7}(r)=r\left(1+2 T \alpha_{5}(r)\right)$ for all $r \in \mathbf{R}^{+}$. Take a radius $r_{1}$ sufficiently small such that the open ball with centre 0 and radius $\alpha_{7}\left(r_{1}\right)$ is a subset of $W$ $\left(B_{\alpha_{7}\left(r_{1}\right)}(0) \subset W\right)$. For each $y \in B_{r_{1}}(0) \subset B_{\alpha_{7}\left(r_{1}\right)}(0) \subset$ $W$ and for all $t \in \mathbf{R}^{+}, y+u(y, t)$ exists and

$$
\|y+u(y, t)\| \leq\|y\|+2 T \alpha_{5}(\|y\|)\|y\|=\alpha_{7}(\|y\|) .
$$

Then for all $t \in \mathbf{R}^{+}$we consider the change of variables $x=y+u(y, t)$ for all $y \in B_{r_{1}}(0): y \in B_{r_{1}}(0)$ implies that $x \in B_{\alpha_{7}\left(r_{1}\right)}(0) \subset W$.

Part VII of the proof will study (21) in more detail showing that (21) is indeed a change of variables and defines a one-to-one relationship.

By differentiating both sides of (21) with respect to $t$, one obtains that for all $y \in B_{r_{1}}(0)$

$$
\dot{x}=\dot{y}+\frac{\partial u}{\partial t}(y, t)+\frac{\partial u}{\partial y}(y, t) \dot{y} .
$$

Since

$$
\dot{x}(t)=f(x(t), t)=f(y(t)+u(y(t), t)),
$$

$$
\begin{gathered}
{\left[I+\frac{\partial u}{\partial y}(y, t)\right] \dot{y}=f(y+u(y, t), t)-\frac{\partial u}{\partial t}(y, t)} \\
{\left[I+\frac{\partial u}{\partial y}(y, t)\right] \dot{y}=f(y+u(y, t), t)-f(y, t)+\bar{f}(y) .}
\end{gathered}
$$

\section{III: Some useful expressions}

In order to prove that the derivative of the Liapunov function $V$ along the trajectories of (26) is negative definite, we first consider some inequalities to be used later on.

Expression (20) implies the existence of a strictly positive $r_{2} \leq r_{1}$ such that for all $y$ with $\|y\|<r_{2}$ (the open ball $B_{r_{2}}(0)$ is sufficiently small: $B_{r_{2}}(0) \subset B_{r_{1}}(0) \subset W$ and $\left.B_{r_{2}}(0) \subset U \subset W\right)$, the matrix

$$
I+\frac{\partial u}{\partial y}(y, t)
$$

is nonsingular and its norm satisfies

$$
\begin{aligned}
& 0<1-2 T \alpha_{5}\left(r_{2}\right)<1-2 T \alpha_{5}(\|y\|) \leq \\
& 1-\left\|\frac{\partial u}{\partial y}(y, t)\right\| \leq\left\|I+\frac{\partial u}{\partial y}(y, t)\right\| .
\end{aligned}
$$

Here $r_{2}<\alpha_{5}^{-1}\left(\frac{1}{2 T}\right)$. Moreover, assuming $y \in B_{r_{2}}(0)$, it can be verified by computation that

$$
\begin{aligned}
& {\left[I+\frac{\partial u}{\partial y}(y, t)\right]^{-1}=} \\
& I-\left[\frac{\partial u}{\partial y}(y, t)-\left(\frac{\partial u}{\partial y}(y, t)\right)^{2}\left(I+\frac{\partial u}{\partial y}(y, t)\right)^{-1}\right]
\end{aligned}
$$

and therefore (with (28))

$$
\begin{gathered}
\left\|\frac{\partial u}{\partial y}(y, t)-\left(\frac{\partial u}{\partial y}(y, t)\right)^{2}\left(I+\frac{\partial u}{\partial y}(y, t)\right)^{-1}\right\| \leq \\
\frac{2 T \alpha_{5}(\|y\|)}{1-2 T \alpha_{5}(\|y\|)} .
\end{gathered}
$$

Even if (27) is a nonsingular matrix, the right hand side of (30) may be large in case $2 T \alpha_{5}(\|y\|)$ approaches 1 . Since $T$ is finite and $\alpha_{5}: \mathbf{R}^{+} \rightarrow \mathbf{R}^{+}$is a class $K$ function, by taking a $r_{3} \leq r_{2}$ sufficiently small $2 T \alpha_{5}(\|y\|)$ can be made arbitrary small when $\|y\|<r_{3}$. In case $2 T \alpha_{5}(\|$ 
$y \|) \leq 0.5$, for all $y$ with $\|y\|<r_{3}$, one obtains that

$$
\left\|\frac{\partial u}{\partial y}(y, t)-\left(\frac{\partial u}{\partial y}(y, t)\right)^{2}\left(I+\frac{\partial u}{\partial y}(y, t)\right)^{-1}\right\| \leq
$$

\section{IV: The evolution of the Liapunov function $V$ along the transformed system}

In order to investigate local uniform asymptotic stability properties, the evolution of the Liapunov function $V$ along the trajectories of the system described by the differential equation (26) is crucial. This leads to the expression (for all $y \in B_{r_{2}}(0) \subset\left(B_{r_{1}}(0) \cap U\right)$ )

$$
\begin{aligned}
\frac{\partial V}{\partial y}(y) \dot{y} & =\frac{\partial V}{\partial y}(y)\left[I+\frac{\partial u}{\partial y}(y, t)\right]^{-1} \\
\cdot[ & {[f(y+u(y, t), t)-f(y, t)+\bar{f}(y)] . }
\end{aligned}
$$

Defining

$$
v(y, t) \triangleq \frac{\partial u}{\partial y}(y, t)-\left(\frac{\partial u}{\partial y}(y, t)\right)^{2}\left(I+\frac{\partial u}{\partial y}(y, t)\right)^{-1}
$$

and invoking (29), we rewrite (32) as (when $y \in B_{r_{2}}(0)$ ),

$$
\frac{\partial V}{\partial y}(y) \dot{y}=\frac{\partial V}{\partial y}(y) \bar{f}(y)+E_{1}(y, t)+E_{2}(y, t)
$$

with

$$
E_{1}(y, t) \triangleq-\frac{\partial V}{\partial y}(y) v(y, t) \bar{f}(y)
$$

$E_{2}(y, t) \triangleq \frac{\partial V}{\partial y}(y)(I-v(y, t))(f(y+u, t)-f(y, t))$.

This implies by (7) that for all $y \in B_{r_{2}}(0)$

$$
\frac{\partial V}{\partial y}(y) \dot{y} \leq-\alpha_{3}(\|y\|)+\left\|E_{1}(y, t)\right\|+\left\|E_{2}(y, t)\right\| .
$$

\section{V: Upper bounds for $\left\|E_{1}(y, t)\right\|$ and $\left\|E_{2}(y, t)\right\|$}

In order to prove that the right hand side of inequality (37) is negative definite, we introduce upper bounds for $\left\|E_{1}(y, t)\right\|$ and $\left\|E_{2}(y, t)\right\|$ that are sufficiently small.

From (8), (31) and (16) one obtains that for all $y$ with $\|y\|<r_{3}$

$$
\left\|E_{1}(y, t)\right\| \leq 4 T \alpha_{4}(\|y\|) \alpha_{5}^{2}(\|y\|)\|y\|
$$

and that

$$
\begin{aligned}
& \left\|E_{2}(y, t)\right\| \leq \\
& \alpha_{4}(\|y\|)\left(1+4 T \alpha_{5}(\|y\|)\right)\|f(y+u, t)-f(y, t)\| .
\end{aligned}
$$

Since for all $y$ with $\|y\|<r_{3}, 2 T \alpha_{5}(\|y\|) \leq 0.5$ and one obtains using (15) that

$$
\left\|E_{2}(y, t)\right\| \leq 2 \alpha_{4}(\|y\|) \quad \alpha_{5}(\max (\|y\|,\|y+u\|))\|u\| .
$$

From (18) and (22), (40) can be rewritten as (for all $y$ with $\left.\|y\|<r_{3}\right)$

$$
\begin{aligned}
& \left\|E_{2}(y, t)\right\| \leq 4 T \alpha_{4}(\|y\|) . \\
& \quad \alpha_{5}\left(\|y\|\left(1+2 T \alpha_{5}(\|y\|)\right)\right) \alpha_{5}(\|y\|)\|y\|
\end{aligned}
$$

It is possible to choose $r_{4}\left(r_{4} \leq r_{3}\right)$ sufficiently small implying that for all y with $\|y\|<r_{4}$

$$
\alpha_{5}\left(\|y\|\left(1+2 T \alpha_{5}(\|y\|)\right)\right) \leq 2 \alpha_{5}(\|y\|) .
$$

This implies that for all $y$ with $\|y\|<r_{4}$

$$
\left\|E_{2}(y, t)\right\| \leq 8 T \alpha_{4}(\|y\|) \alpha_{5}^{2}(\|y\|)\|y\| .
$$

\section{VI: Uniform asymptotic stability in the new co- ordinates}

We are now ready to prove that the equilibrium point of (26) (the system obtained after performing the change of variables) is locally uniformly asymptotically stable by proving that the right hand side of (37) is negative definite. By combining (37), (38) and (43), one obtains that for all $y$ with $\|y\|<r_{4}$

$$
\begin{gathered}
\frac{\partial V}{\partial y}(y) \dot{y} \leq-\alpha_{3}(\|y\|)+12 T \alpha_{4}(\|y\|) \alpha_{5}^{2}(\|y\|)\|y\| \\
\frac{\partial V}{\partial y}(y) \dot{y} \leq-\alpha_{3}(\|y\|)\left(1-12 T \alpha_{6}(\|y\|)\right) .
\end{gathered}
$$

A crucial role is played by the technical assumption (10) on $\alpha_{6}$ : since $\alpha_{6}$ is a class $K$ function, it is possible to choose a $r_{5}\left(r_{5}<r_{4}\right)$ such that for all $y$ with $\|y\| \leq r_{5}$

$$
\alpha_{6}(\|y\|) \leq \frac{1}{24 T}
$$

and

$$
\frac{\partial V}{\partial y}(y) \dot{y} \leq-0.5 \alpha_{3}(\|y\|)
$$

Expression (47) implies local uniform asymptotic stability of the equilibrium point $y=0$ of the system (26) with differential equation 
$\dot{y}=\left[I+\frac{\partial u}{\partial y}(y, t)\right]^{-1}(f(y+u(y, t), t)-f(y, t)+\bar{f}(y))$.

Since (47) is satisfied for all $y$ with $\|y\| \leq r_{5}$, it follows that $\left\|y\left(t, t_{0}, y_{0}\right)\right\| \leq r_{5}$ for all $t_{0} \in \mathbf{R}^{+}$, for all $t \geq t_{0}$ and for all $y_{0}$ with $\left\|y_{0}\right\| \leq r_{6}=\alpha_{2}^{-1}\left(\alpha_{1}\left(r_{5}\right)\right)$. Moreover, there exists a class $K L$ function $\beta$ such that for all $y_{0}$ with $\left\|y_{0}\right\| \leq r_{6}=\alpha_{2}^{-1}\left(\alpha_{1}\left(r_{5}\right)\right)$, for all $t_{0} \in \mathbf{R}^{+}$and for all $t \geq t_{0}([5]$, p. 152, Theorem 4.9),

$$
\left\|y\left(t, t_{0}, y_{0}\right)\right\| \leq \beta\left(\left\|y_{0}\right\|, t-t_{0}\right)
$$

$y\left(t, t_{0}, y_{0}\right)$ denotes the solution of the differential equation (26) (i.e. (48)) at $t$ with initial condition $y_{0}$ at $t_{0}$ (or $y(t)$ for short). The function $\beta:[0, a) \times \mathbf{R}^{+} \rightarrow \mathbf{R}^{+}$(for some $a>0)$ is a class $K L$ function.

\section{VII: The function $x=y+u(y, t)$ defines a one-to- one relationship locally}

With the help of transformation (21), we will show that local uniform asymptotic stability of $y=0$ of (26) (i.e. (48)) implies local uniform asymptotic stability of $x=0$ of (1). In order to prove that the evolution of all the trajectories of (1) are bounded by class KL functions, it is sufficient to show that

- there exists a neighbourhood of $x=0$ such that for all $x_{0}$ in this neighbourhood and for all $t_{0}$ there exists a unique $y_{0}$ such that $x_{0}=y_{0}+u\left(y_{0}, t_{0}\right)$ (see the current part VII of the proof)

- the trajectory $y\left(t, t_{0}, y_{0}\right)$ of (26) (i.e. (48)) starting at $y_{0}$ at $t_{0}$ corresponds with the trajectory $x\left(t, t_{0}, x_{0}\right)$ of (1) (see part VIII of the proof)

- the boundedness of $\left\|y\left(t, t_{0}, y_{0}\right)\right\|$ by a class KL function implies boundedness of $\left\|x\left(t, t_{0}, x_{0}\right)\right\|$ by a class KL function (see part VIII of the proof).

Consider the function described by $x=y+u(y, t)$. Since $f(y, t)$ is continuously differentiable with respect to $y$, the functions $\bar{f}(y), h(y, t)$ and $u(y, t)$ are also continuously differentiable with respect to $y$ (for each fixed $t$ ). These continuous differentiability properties hold in $W$ and therefore also in the open ball $B_{r_{2}}(0)$ and the open ball $B_{r_{5}}(0)$. Notice that $0+u(0, t)=0$ for all $t \in \mathbf{R}^{+}$.

We now prove that $x=y+u(y, t)$ defines a one-to-one relationship. It is clear that every $y$ and every $t$ define one $x$; we will also prove that each $x$ and $t$ define one $y$. First we show that to each $x$ and $t$ there corresponds at least one $y$.

Expression (27) is the Jacobian matrix of $y+u(y, t)$, which is nonsingular in $B_{r_{2}}(0)$; the Jacobian determinant (i.e. the determinant of (27)) is nonzero for all $y$ in $B_{r_{2}}(0)$.
Using (21) and (18), for all $y \in B_{r_{2}}(0)$ and for all $t \in \mathbf{R}^{+}$

$$
\begin{gathered}
\|y\|\left(1-2 T \alpha_{5}(\|y\|)\right) \leq\|y+u(y, t)\| \leq \\
\|y\|\left(1+2 T \alpha_{5}(\|y\|)\right) .
\end{gathered}
$$

For all $y$ with $\|y\|<r_{3}, 2 T \alpha_{5}(\|y\|) \leq 0.5$ which implies that for all $t \in \mathbf{R}^{+}$and for all $\|y\|<r_{3} \leq r_{2}$

$$
0.5\|y\| \leq\|y+u(y, t)\| \leq 1.5\|y\| .
$$

Since $r_{6} \leq r_{5}<r_{4} \leq r_{3}$, this implies that $\|y+u(y, t)\|$ is strictly positive on $S_{r_{6}}(0)$ (defined as the set of all $y$ with $\left.\|y\|=r_{6}\right)$. The minimum of the continuous function $\|y+u(y, t)\|$ on the compact set $S_{r_{6}}(0)$ equals $m(t)$ and $m(t) \geq 0.5 r_{6}>0$ for all $t \in \mathbf{R}^{+}$.

Consider now the open ball $B_{0.25 r_{6}}(0)$ with radius $0.25 r_{6}$. We will prove that for each point $x \in B_{0.25 r_{6}}(0)$ and for each $t$, there exists a point $y \in\left(B_{r_{6}}(0) \cup S_{r_{6}}(0)\right)$ such that $x=y+u(y, t)$.

Choose $x \in B_{0.25 r_{6}}(0)$ (and keep $x$ fixed). Define for each $y \in\left(B_{r_{6}}(0) \cup S_{r_{6}}(0)\right)$ and for every $t \in \mathbf{R}^{+}$, the real valued function

$$
k(y, t)=\|y+u(y, t)-x\| .
$$

Notice that for every $t \in \mathbf{R}^{+}$the function $k(y, t)$ is continuous in $y$ and attains a minimum on the compact set $\left(B_{r_{6}}(0) \cup S_{r_{6}}(0)\right)$. We will show that for every $t, k(y, t)$ attains this minimum somewhere in $B_{r_{6}}(0)$. At $y=0$, we have $k(0, t)=\|x\|<0.25 r_{6} \leq m(t) / 2$. This implies that the minimum of $k(y, t)$ must be strictly smaller than $0.25 r_{6} \leq m(t) / 2$. At each point $y$ of $S_{r_{6}}(0)$, using (52) we have

$$
\begin{aligned}
& k(y, t) \geq\|y+u(y, t)\|-\|x\|> \\
& \|y+u(y, t)\|-0.25 r_{6} \geq 0.25 r_{6} .
\end{aligned}
$$

Since the minimum of $k(y, t)$ on $B_{r_{6}}(0)$ is strictly smaller than $0.25 r_{6}$ and since the minimum of $k(y, t)$ on $S_{r_{6}}(0)$ is $\geq 0.25 r_{6}$, the minimum of $k(y, t)$ on $B_{r_{6}}(0) \cup S_{r_{6}}(0)$ will be attained in $B_{r_{6}}(0)$. Hence, there is an interior point $c$ in $B_{r_{6}}(0)$ at which $k(y, t)$ attains its minimum which we will prove to be equal to 0 . At this point, $k^{2}(y, t)$ has also a minimum with

$k^{2}(y, t)=\|y+u(y, t)-x\|^{2}=\sum_{r=1}^{n}\left(y_{r}+u_{r}(y, t)-x_{r}\right)^{2}$.

Since each partial derivative of $k^{2}(y, t)$ must be zero at $c$, it follows that for each $k \in\{1, \ldots, n\}$ ([2], p. 370)

$$
\left.2 \sum_{r=1}^{n} \frac{\partial\left(y_{r}+u_{r}(y, t)\right)}{\partial y_{k}}\left(y_{r}+u_{r}(y, t)-x_{r}\right)\right|_{y=c}=0 .
$$


Since (55) is a system of linear equations with nonzero determinant (i.e. the non-singular Jacobian determinant of $y+u(y, t))$, one obtains that

$$
\left.\left(y_{r}+u_{r}(y, t)-x_{r}\right)\right|_{y=c}=0
$$

for each $r$. This implies that $c+u(c, t)=x$. This establishes that for every $x \in B_{0.25 r_{6}}(0)$ and for every $t$, there exists an $y \in B_{r_{6}}(0)$ such that $y+u(y, t)=x$.

We now show that there is only one $y$ satisfying this condition. Suppose there exists a $y_{1} \in B_{r_{6}}(0)$ and a different $y_{2} \in B_{r_{6}}(0)$ such that $x=y_{1}+u\left(y_{1}, t\right)=$ $y_{2}+u\left(y_{2}, t\right)$. This implies that

$$
0=y_{1}-y_{2}+u\left(y_{1}, t\right)-u\left(y_{2}, t\right)
$$

and by (13)

$$
\begin{gathered}
\left\|y_{1}-y_{2}\right\|=\left\|u\left(y_{1}, t\right)-u\left(y_{2}, t\right)\right\| \leq \\
\left\|\int_{0}^{t}\left(\left(f\left(y_{1}, \tau\right)-f\left(y_{2}, \tau\right)\right)-\left(\bar{f}\left(y_{1}\right)-\bar{f}\left(y_{2}\right)\right)\right) d \tau\right\| .
\end{gathered}
$$

Using (15), this implies that

$$
\begin{array}{r}
\left\|y_{1}-y_{2}\right\| \leq 2 T \max \left(\alpha_{5}\left(\left\|y_{1}\right\|\right), \alpha_{5}\left(\left\|y_{2}\right\|\right)\right)\left\|y_{1}-y_{2}\right\| \leq \\
2 T \alpha_{5}\left(r_{6}\right)\left\|y_{1}-y_{2}\right\| .
\end{array}
$$

Since $r_{6} \leq r_{5}<r_{4} \leq r_{3}, 2 T \alpha_{5}\left(r_{6}\right) \leq 0.5$ leading to the contradiction $\left\|y_{1}-y_{2}\right\| \leq 0.5\left\|y_{1}-y_{2}\right\|$. So, for every $t \in \mathbf{R}^{+}$and $x \in B_{0.25 r_{6}}(0)$, there exists one and only one $y \in B_{r_{6}}(0)$ such that $y+u(y, t)=x$. This implies $y+$ $u(y, t)=x$ indeed defines a one-to-one relation locally.

\section{VIII: Uniform asymptotic stability of the original system (1)}

Formulating the local uniform asymptotic stability property of the equilibrium point of (26) using class KL functions, it is now possible to prove that also the evolution of the trajectories of (1) are bounded by class KL functions. This implies local uniform asymptotic stability of the equilibrium point of (1). More precisely, it is sufficient

- to show that the trajectory $y\left(t, t_{0}, y_{0}\right)$ of (26) (i.e. (48)) starting at $y_{0}$ at $t_{0}$ corresponds with the trajectory $x\left(t, t_{0}, x_{0}\right)$ of $(1)$

- to show that boundedness of $\left\|y\left(t, t_{0}, y_{0}\right)\right\|$ by a class KL function implies boundedness of $\left\|x\left(t, t_{0}, x_{0}\right)\right\|$ by a class KL function.

First, with an arbitrary $x_{0} \in B_{0.25 r_{6}}(0)$ and $t_{0} \in \mathbf{R}^{+}$, there corresponds one and only one $y_{0} \in B_{r_{6}}(0)$ such that $y_{0}+u\left(y_{0}, t_{0}\right)=x_{0}$. Consider the trajectory $y\left(t, t_{0}, y_{0}\right)$ of (26) (i.e. (48)) satisfying (49) with $\left\|y\left(t, t_{0}, y_{0}\right)\right\| \leq r_{5}<r_{3} \leq r_{1}$ implying that $2 T \alpha_{5}\left(\left\|y\left(t, t_{0}, y_{0}\right)\right\|\right) \leq 0.5$. The transformation (21) defines a corresponding function $y\left(t, t_{0}, y_{0}\right)+u\left(y\left(t, t_{0}, y_{0}\right), t\right)$. Taking its derivative with respect to time and using $(26)$, one verifies that $y\left(t, t_{0}, y_{0}\right)+u\left(y\left(t, t_{0}, y_{0}\right), t\right)=$ $x\left(t, t_{0}, x_{0}\right)$ where $x\left(t, t_{0}, x_{0}\right)$ is a solution of $(1)$.

Secondly, from (21) and (18), for all $y \in B_{r_{2}}(0)$ with the corresponding $x$

$$
\|y\|\left(1-2 T \alpha_{5}(\|y\|)\right) \leq\|x\| \leq\|y\|\left(1+2 T \alpha_{5}(\|y\|)\right) .
$$

Since $2 T \alpha_{5}\left(\left\|y\left(t, t_{0}, y_{0}\right)\right\|\right) \leq 0.5$ in case $\left\|y\left(t, t_{0}, y_{0}\right)\right\| \leq$ $r_{5}$, one obtains that

$$
0.5\left\|y\left(t, t_{0}, y_{0}\right)\right\| \leq\left\|x\left(t, t_{0}, x_{0}\right)\right\| \leq 1.5\left\|y\left(t, t_{0}, y_{0}\right)\right\| .
$$

Relying on the state transformation (21), the conditions (49) and (61) imply that the solution of the system (1) satisfies the inequality

$$
\left\|x\left(t, t_{0}, x_{0}\right)\right\| \leq 1.5 \beta\left(2\left\|x_{0}\right\|, t-t_{0}\right) .
$$

The inequality (62) is valid for all $t_{0} \in \mathbf{R}^{+}$, for all $t \geq t_{0}$ and for all $x_{0}$ with $\left\|x_{0}\right\|<0.25 r_{6}$. Since the right hand side of (62) is a class $K L$ function, (62) implies local uniform asymptotic stability of the equilibrium point $x=0$ of the system (1).

\section{The main application and examples}

In this section we introduce a class of systems fitting the assumptions of the main theorem. This in turn leads to a number of examples. There is no homogeneity assumption, neither is there any assumption on the timevariance of the vectorfield.

Consider the time-varying periodic system

$$
\dot{x}(t)=f_{1}(x(t), t)
$$

which satisfies the properties listed for (1), except for (9).

For all $x \in W$, define the averaged system

$$
\dot{x}(t)=\bar{f}_{1}(x(t))
$$

where

$$
\bar{f}_{1}(x)=\frac{1}{T} \int_{0}^{T} f_{1}(x, t) d t .
$$

Assume that $f_{1}$ is continuously differentiable with respect to $x$ and the Jacobian matrix $\left[\partial f_{1} / \partial x\right]$ is bounded on $W$, uniformly in $t$. Then also $\bar{f}_{1}$ is continuously differentiable with respect to $x$ on $W$ and its Jacobian matrix $\left[\partial \bar{f}_{1} / \partial x\right]$ is bounded on $W$. 
The equilibrium point $x=0$ of the averaged system (64) is assumed to be exponentially stable. Let $k_{1}, \lambda_{1}$ and $r_{1}$ be positive constants. The open ball $B_{k_{1} r_{1}}(0) \subset W$. For all $x_{0} \in B_{r_{1}}(0)$, for all $t_{0} \in \mathbf{R}^{+}$and for all $t \geq t_{0}$, the trajectories of (64) satisfy

$$
\left\|x\left(t, t_{0}, x_{0}\right)\right\| \leq k_{1}\left\|x_{0}\right\| e^{-\lambda_{1}\left(t-t_{0}\right)} .
$$

It follows from a Liapunov converse theorem ([5], Theorem 4.14), that there exists a Liapunov function $V$ : $B_{r_{1}}(0) \rightarrow \mathbf{R}$ such that

$$
\begin{gathered}
c_{1}\|x\|^{2} \leq V(x) \leq c_{2}\|x\|^{2}, \\
\frac{\partial V}{\partial x}(x) \bar{f}_{1}(x) \leq-c_{3}\|x\|^{2}, \\
\left\|\frac{\partial V}{\partial x}(x)\right\| \leq c_{4}\|x\|,
\end{gathered}
$$

for some strictly positive constants $c_{1}, c_{2}, c_{3}$ and $c_{4}$.

\subsection{Main Application}

Consider a continuous positive definite function $h: W \rightarrow \mathbf{R}$ for which there exist class $\mathrm{K}$ functions $\alpha_{h 1}, \alpha_{h 2}: \mathbf{R}^{+} \rightarrow \mathbf{R}^{+}$such that for all $x \in W:$

$$
\alpha_{h 1}(\|x\|) \leq h(x) \leq \alpha_{h 2}(\|x\|) .
$$

Moreover, assume there exists a strictly positive constant $c_{5}$ such that for all $x \in W$,

$$
\left\|\frac{\partial h}{\partial x}(x)\right\| \cdot\|x\| \leq c_{5} \alpha_{h 2}(\|x\|) .
$$

Assume further that $\alpha_{h 2}: \mathbf{R}^{+} \rightarrow \mathbf{R}^{+}$has the additional property that sufficiently close to the origin $\alpha_{h 2}\left(\|x\|\left(1+2 T \alpha_{h 2}(\|x\|)\right)\right) \leq 2 \alpha_{h 2}(\|x\|)$. For all $x \in W$ and for all $t \in \mathbf{R}^{+}$, define the time-varying system

$$
\dot{x}(t)=f_{2}(x(t), t) \triangleq h(x(t)) f_{1}(x(t), t) .
$$

We assume that $h(x) f_{1}(x, t)$ is continuously differentiable with respect to $x$ on $W$ for all $t \in \mathbf{R}^{+}$.

Assume that

$$
\|x\| \mapsto \frac{\alpha_{h 2}^{2}(\|x\|)}{\alpha_{h 1}(\|x\|)}
$$

is a class $\mathrm{K}$ function.

Typical candidates for $h$ are $h(x)=x^{T} P x, h(x)=$ $\|x\|^{g(x)}$ or $h(x)=\|x\|^{\beta}$ with $\beta$ constant. This will be discussed in more detail in the following sections.

We will show-based on the main theorem and the existence of a Liapunov function $V$ - that the equilibrium point $x=0$ of the differential equation (72) is locally uniformly asymptotically stable.

\section{Proof:}

The system (72) is time-periodic with period $T$. Invoking $V$, we will first prove asymptotic stability of the origin of

$$
\dot{x}(t)=\bar{f}_{2}(x(t)) \triangleq h(x(t)) \bar{f}_{1}(x(t))
$$

which is the averaged system of (72). The Liapunov function $V$ satisfies (6) and (7). By setting $U=B_{r_{1}}(0)$, $\alpha_{1}(\|x\|)=c_{1}\|x\|^{2}$ and $\alpha_{2}(\|x\|)=c_{2}\|x\|^{2}$, (6) is satisfied. Using (68)

$$
\frac{\partial V}{\partial x}(x) \cdot h(x) \cdot \bar{f}_{1}(x) \leq-c_{3} h(x)\|x\|^{2} \leq-c_{3} \alpha_{h 1}(\|x\|)\|x\|^{2}
$$

and by setting $\alpha_{3}(\|x\|)=c_{3} \alpha_{h 1}(\|x\|)\|x\|^{2}$ with $U=$ $B_{r_{1}}(0)(7)$ is satisfied. This implies asymptotic stability of the equilibrium point of (74).

We will now verify conditions (8), (9) and (10) which implies local uniform asymptotic stability of the equilibrium point of (72) because of the main theorem.

Considering (69) with $U=B_{r_{1}}(0)$, (8) is satisfied by setting $\alpha_{4}(\|x\|)=c_{4}\|x\|$. Since $f_{2}(x, t)=h(x) f_{1}(x, t)$ is continuously differentiable with respect to $x$ on $W$ for all $t \in \mathbf{R}^{+}$,

$$
\left\|\frac{\partial f_{2}}{\partial x}(x, t)\right\| \leq\left\|\frac{\partial h}{\partial x}(x)\right\| \cdot\left\|f_{1}(x, t)\right\|+\|h(x)\| \cdot\left\|\frac{\partial f_{1}}{\partial x}(x, t)\right\| .
$$

Since $f_{1}$ is continuously differentiable with respect to $x$ and the Jacobian matrix is bounded on $W$, uniformly in $t$, there exists a bound $c_{6}>0$ such that for all $x \in W$ and for all $t \in \mathbf{R}^{+}$:

$$
\left\|\frac{\partial f_{1}}{\partial x}(x, t)\right\| \leq c_{6}
$$

Then ( cfr. Lemma 3.1 on pp. 89-90 [5]), one obtains that for all $x \in W$ and for all $t \in \mathbf{R}^{+}$that $\left\|f_{1}(x, t)\right\| \leq$ $c_{6}\|x\|$. From (76), together with (70) and (77), one obtains that

$$
\left\|\frac{\partial f_{2}}{\partial x}(x, t)\right\| \leq\left\|\frac{\partial h}{\partial x}(x)\right\| c_{6}\|x\|+c_{6} \alpha_{h 2}(\|x\|) .
$$

From (71), the following is true on $W$

$$
\left\|\frac{\partial f_{2}}{\partial x}(x, t)\right\| \leq c_{6}\left(1+c_{5}\right) \alpha_{h 2}(\|x\|),
$$

such that (9) is satisfied by taking $\alpha_{5}(\|x\|)=$ $c_{6}\left(1+c_{5}\right) \alpha_{h 2}(\|x\|)$. This definition implies that sufficiently close to the origin $\alpha_{5}\left(\|x\|\left(1+2 T \alpha_{5}(\|x\|)\right)\right) \leq$ $2 \alpha_{5}(\|x\|)$. 
Finally (with an appropriate choice of $c_{7}>0$ ),

$$
\frac{\alpha_{4}(\|x\|) \alpha_{5}^{2}(\|x\|)\|x\|}{\alpha_{3}(\|x\|)}=c_{7} \frac{\alpha_{h 2}^{2}(\|x\|)}{\alpha_{h 1}(\|x\|)}
$$

such that also (10) is satisfied. This is based on the property that (73) is a class $\mathrm{K}$ function.

All the conditions required by the main theorem are satisfied; therefore the equilibrium point $x=0$ of (72) is locally uniformly asymptotically stable.

Remark 4: The main application requires the timevarying system (63) to have an averaged system (64) with a locally exponentially stable equilibrium point. The positive definite function $h$ satisfying (70) and (71) is crucial: it guarantees that close to the equilibrium point, trajectories of (72) are slowly varying in comparison with the vectorfield itself. Actually, $h$ plays a role similar to the role played by the parameter $\epsilon$ in the proof that exponential stability of the averaged system $\dot{x}(t)=\varepsilon \bar{f}(x(t))$ implies exponential stability of the original time-varying system $\dot{x}(t)=\varepsilon f(x(t), t)[1,5]$. Notice that because of (70), this classical case is not covered by the main application, since a constant $\epsilon$ does not qualify as a particular case of $h$. This implies also that e.g. the pendulum discussed in [5], example 10.10, is not covered by the results presented in this paper. The reader is referred to Remark 3 for a related remark.

Remark 5: Although extensions relaxing (71) and (73) may be possible, some constraints will be required. Notice that by (70) and (73), the upper and lower bounds of $h$ are related. This defines a sector constraining $h$. Within this sector, (71) imposes additional restrictions on $h$ by putting constraints on its derivative.

Remark 6: The formulation and proof of the main application involve three different systems. First, exponential stability of (64) implies asymptotic stability of the time invariant system (74). Since (74) is the averaged system of (72), local uniform asymptotic stability of the original time-varying system (72) follows from the main theorem. Notice that the vectorfield (72) is not fast timevarying.

\subsection{Nonhomogeneous examples}

In this section we discuss some examples, obtained as special cases of the main application presented in the previous section. They correspond to a specific choice of $h$. Notice that the vectorfields involved are not homogeneous: their stability properties cannot be established based on the results presented in [8].

Example 1: Consider the time-varying periodic system (63) defined in the previous section. The averaged system (64) is supposed to satisfy all the conditions required in the previous section and has an exponentially stable equilibrium point at the origin. Consider for all $x \in W$ the quadratic function $x^{T} P x$, with $P$ a positive definite matrix. Choose $h(x)=x^{T} P x$, then equation (72) becomes for all $x \in W$ and for all $t \in \mathbf{R}^{+}$

$$
\dot{x}(t)=f_{2}(x(t), t)=x(t)^{T} P x(t) f_{1}(x(t), t) .
$$

Local uniform asymptotic stability of the equilibrium point $x=0$ of (81) follows from the main application.

\section{Proof:}

The conditions imposed by the main application are satisfied. Taking $\alpha_{h 1}(\|x\|)=\lambda_{\min }(P)\|x\|^{2}, \alpha_{h 2}(\|x\|)=$ $\lambda_{\max }(P)\|x\|^{2},(70)$ is satisfied. Expression (71) is satisfied with $c_{5}=2$.

Since $\alpha_{h 2}(\|x\|)=\lambda_{\max }(P)\|x\|^{2}$, it follows that $\alpha_{h 2}\left(\|x\|\left(1+2 T \alpha_{h 2}(\|x\|)\right)\right) \leq 2 \alpha_{h 2}(\|x\|)$ with

$$
\|x\| \leq \sqrt{\frac{\sqrt{2}-1}{2 T \lambda_{\max }(P)}} .
$$

The conditions imposed on $f_{1}(x, t)$ imply that $x^{T} P x f_{1}(x, t)$ is continuously differentiable with respect to $x$ on $W$ for all $t \in \mathbf{R}^{+}$. Since

$$
\frac{\alpha_{h 2}^{2}(\|x\|)}{\alpha_{h 1}(\|x\|)}=\frac{\lambda_{\max }^{2}(P)}{\lambda_{\min }(P)}\|x\|^{2}
$$

is a class $\mathrm{K}$ function, local uniform asymptotic stability of the equilibrium point $x=0$ of (81) follows from the main application.

Example 2: Consider the nonhomogeneous timevarying system

$$
\dot{x}(t)=A(t) x(t)\|x(t)\|^{g(x(t))}
$$

defined on some open and convex subset $W \subset$ $B_{1}(0) \subset \mathbf{R}^{n}(0 \in W)$. The exponent $g$ is continuously differentiable with respect to $x$ on $W$. For all $x \in W: 0<g_{2} \leq g(x) \leq g_{1}$ (both $g_{1}$ and $g_{2}$ are real numbers).

There exists an $A_{M}>0$ such that for all $t \in \mathbf{R}^{+}$: $\|A(t)\| \leq A_{M}$. Consider the case where the $n \times n$ matrix $A(t)$ is time-periodic with period $T$ and

$$
\bar{A}=\frac{1}{T} \int_{0}^{T} A(t) d t
$$

is Hurwitz. When for all $x \in W$

$$
\left\|\frac{\partial g}{\partial x}(x)\right\| \leq g_{3},
$$


where $g_{3} \in \mathbf{R}$ and $2 g_{2}>g_{1}$, local uniform asymptotic stability of the equilibrium point $x=0$ of (84) is a consequence of the main application.

Proof: Basically, the proof is a verification of the conditions listed in the main application, after introducing appropriate choices of $f_{1}$ and $h$, given by (87) and (89) respectively. Other choices do not fit the main application. Consider

$$
\dot{x}(t)=f_{1}(x(t), t)=A(t) x(t),
$$

and its corresponding averaged system

$$
\dot{x}(t)=\bar{f}_{1}(x(t))=\bar{A} x(t) .
$$

The vector fields $f_{1}$ and $\bar{f}_{1}$ are defined on $\mathbf{R}^{n}$ and therefore also on $W \subset B_{1}(0) \subset \mathbf{R}^{n} ; f_{1}$ is continuously differentiable with respect to $x$ and the Jacobian matrix $\left[\partial f_{1} / \partial x\right]=A(t)$ with $\|A(t)\| \leq A_{M}$ for all $t$. Moreover, $\left[\partial \bar{f}_{1} / \partial x\right]=\bar{A}$ and $\|\bar{A}\| \leq A_{M}$.

The averaged system (88) is globally exponentially stable. The scalar function $h$ defined by

$$
h(x)=\|x\|^{g(x)}
$$

for all $x \in W \subset B_{1}(0)$, satisfies (70) with $\alpha_{h 1}(\|x\|)=$ $\|x\|^{g_{1}}$ and $\alpha_{h 2}(\|x\|)=\|x\|^{g_{2}}$.

Since

$$
\begin{aligned}
\frac{\partial h}{\partial x}(x)=\frac{\partial}{\partial x}\left(\|x\|^{g(x)}\right) & =g(x)\|x\|^{g(x)-1} \frac{\partial}{\partial x}(\|x\|)(90) \\
& +\|x\|^{g(x)} \ln (\|x\|) \frac{\partial}{\partial x}(g(x))
\end{aligned}
$$

one obtains that

$$
\begin{aligned}
\left\|\frac{\partial h}{\partial x}(x)\right\|\|x\| & \leq g(x)\|x\|^{g(x)}\left\|\frac{\partial}{\partial x}(\|x\|)\right\|+ \\
& +\|x\|^{g(x)+1}|\ln (\|x\|)|\left\|\frac{\partial g}{\partial x}(x)\right\| .
\end{aligned}
$$

For all $x \in W \subset B_{1}(0)$,

$$
\left\|\frac{\partial h}{\partial x}(x)\right\|\|x\| \leq\|x\|^{g_{2}}\left(g_{1}+\|x\||\ln (\|x\|)| g_{3}\right)
$$

For all $x$ with $\|x\|<1,\|x\| \cdot|\ln (\| x \mid)|$ reaches its maximum value when $\|x\|=e^{-1}$ implying that $\|x\| \cdot|\ln (\|x\|)| \leq e^{-1}$. It follows that

$\left\|\frac{\partial h}{\partial x}(x)\right\|\|x\| \leq\left(g_{1}+g_{3} e^{-1}\right)\|x\|^{g_{2}}=c_{5} \alpha_{h 2}(\|x\|)$. with $c_{5}=g_{1}+g_{3} e^{-1}$. This implies that condition (71) is satisfied. Moreover, taking

$$
\|x\| \leq(2 T)^{-\frac{1}{g_{2}}}\left(2^{\frac{1}{g_{2}}}-1\right)^{\frac{1}{g_{2}}}
$$

one obtains that $\alpha_{h 2}\left(\|x\|\left(1+2 T \alpha_{h 2}(\|x\|)\right)\right) \leq$ $2 \alpha_{h 2}(\|x\|)$.

Since (73) can be written as

$$
\frac{\alpha_{h 2}^{2}(\|x\|)}{\alpha_{h 1}(\|x\|)}=\|x\|^{2 g_{2}-g_{1}}
$$

the assumption that $2 g_{2}>g_{1}$ implies that the function defined by (95) is a class $\mathrm{K}$ function. Local uniform asymptotic stability of the equilibrium point $x=0$ of (84) follows from the main application.

Remark 7: In case $g_{1}=g_{2}>0$, (84) describes a homogeneous system of positive order. It is shown in [8] that asymptotic stability of the averaged homogeneous system implies local uniform asymptotic stability of the original time-varying homogeneous system when the order is strictly positive. Example 2 (where $g_{1}$ and $g_{2}$ may be different) illustrates that the main theorem and the main application generalise the results formulated in [8]. Notice further that the homogeneous case can also be handled by the current results as will be illustrated in section 3.3.

Remark 8: The stability result of Example 2 is based on the main application. It is possible to prove the same result by an immediate use of the main theorem using the uniform asymptotic stability property of the averaged system

$$
\dot{x}(t)=\bar{A} x(t)\|x(t)\|^{g(x(t))} .
$$

Remark 9: The function $h$ in Example 1 and in Example 2 is such that $h(x)=x^{T} P x$, resp. $h(x)=\|x\|^{g(x)}$. Assuming that (71) is satisfied, a simple additional example is obtained by letting $h$ to be a class $\mathrm{K}$ function $\alpha$, i.e. for all $x \in W: h(x)=\alpha(\|x\|)$. This implies that $\alpha_{h 1}(\|x\|)=\alpha_{h 2}(\|x\|)=\alpha(\|x\|)$ and that (73) is satisfied.

Remark 10: Additional choices for $h$ are possible. We introduce a class of nonhomogeneous functions $h$. Let the real numbers $p_{i}>1$ for all $i \in\{1, \ldots, n\}$. Let $h$ be

$$
h(x)=\left|x_{1}\right|^{p_{1}}+\ldots+\left|x_{n}\right|^{p_{n}} .
$$

Here, $x=\left(x_{1}, \ldots, x_{2}\right)^{T}$ and we restrict $x$ to values with $\|x\|<1$ (Euclidean norm). Let $p_{\text {min }}$ be the minimum of all $p_{i}$, and $p_{\max }$ be the maximum of all $p_{i}$. It is clear that

$\left|x_{1}\right|^{p_{\max }}+\ldots+\left|x_{n}\right|^{p_{\max }} \leq h(x) \leq\left|x_{1}\right|^{p_{\min }}+\ldots+\left|x_{n}\right|^{p_{\min }}$. 
Denote $\|x\|_{p_{\max }}$ as the $p_{\max }$-norm and $\|x\|_{p_{\min }}$ as the $p_{\text {min }}$-norm. ${ }^{1}$ By equivalence of norms, there exist $C_{1}>$ 0 and $C_{2}>0$ such that for all $x: C_{1}\|x\| \leq\|x\|_{p_{\max }}$ and $\|x\|_{p_{\min }} \leq C_{2}\|x\|$. From (98), it follows that

$$
\begin{aligned}
& C_{1}^{p_{\max }}\|x\|^{p_{\max }} \leq \\
& \|x\|_{p_{\max }}^{p_{\max }} \leq h(x) \leq\|x\|_{p_{\min }}^{p_{\min }} \leq \\
& C_{2}^{p_{\min }}\|x\|^{p_{\min }} .
\end{aligned}
$$

Taking $\alpha_{h 1}(\|x\|)=C_{1}^{p_{\max }}\|x\|^{p_{\max }}$ and $\alpha_{h 2}(\|x\|)=$ $C_{2}^{p_{\min }}\|x\|^{p_{\min }},(70)$ is satisfied. In case $2 p_{\min }>p_{\max }$, also (73) is satisfied. We now show that (71) is also satisfied. Indeed,

$$
\begin{array}{r}
\left\|\frac{\partial h}{\partial x}(x)\right\|_{\infty}=\max \left\{p_{1}\left|x_{1}\right|^{p_{1}-1}, \ldots, p_{n}\left|x_{n}\right|^{p_{n}-1}\right\} \leq \\
p_{\max }\|x\|^{p_{\min }-1} .
\end{array}
$$

We used the property that for all $i \in\{1, \ldots, n\}:\left|x_{i}\right| \leq$ $\|x\|$. Since for all $x,\|x\| \leq \sqrt{n}\|x\|_{\infty},(100)$ implies that

$$
\left\|\frac{\partial h}{\partial x}(x)\right\| \cdot\|x\| \leq \sqrt{n} p_{\max }\|x\|^{p_{\min }} .
$$

Taking $c_{5}=\sqrt{n} p_{\max } / C_{2}^{p_{\min }},(71)$ is satisfied.

\subsection{Homogeneous examples}

When reconsidering Example 2 with the additional assumption that $g_{1}=g_{2}$, a homogeneous system is obtained and the main application may be invoked to establish uniform asymptotic stability properties for systems covered before (cfr. [8]). This is illustrated in Example 3 and Example 4.

Example 3: Consider for all $x \in W$ the homogeneous time-varying system $\left(W \subset B_{1}(0) \subset \mathbf{R}^{n}, W\right.$ is an open and convex set containing the origin)

$$
\dot{x}(t)=A(t) x(t)\|x(t)\|^{\beta}
$$

with $\beta>0$. Let the matrix $A(t)$ be time-periodic with period $T$ and let

$$
\bar{A}=\frac{1}{T} \int_{0}^{T} A(t) d t
$$

be Hurwitz. Furthermore, there exists a $A_{M}>0$ such that for all $t \in \mathbf{R}^{+}:\|A(t)\| \leq A_{M}$.

The conditions listed in Example 2 are satisfied ( taking $g_{1}=g_{2}=g(x)=\beta$ ); therefore we obtain local uniform

\footnotetext{
1 The $p$-norm of $x$ is defined as $\|x\|_{p}=$ $\left(\left|x_{1}\right|^{p}+\ldots+\left|x_{n}\right|^{p}\right)^{1 / p}$. In case $p=2$, the Euclidean norm is obtained.
}

asymptotic stability of the equilibrium point $x=0$ of (102).

Remark 11: When $\beta=0$, we obtain a linear system and the conditions of Example 2, the main application and the main theorem are no longer satisfied $\left(0<g_{2} \leq g(x)\right.$ is not true implying that conditions (9) and (10) are not satisfied). The main theorem does not prove uniform asymptotic stability of the original time-varying system. Based on [1,5], stability results may be derived when the vectorfield is fast time-varying.

Remark 12: The stability result of Example 3 is based on Example 2 and the main application. It is possible to prove the same result directly from the main theorem, assuming uniform asymptotic stability property of the origin of the averaged system

$$
\dot{x}(t)=\bar{A} x(t)\|x(t)\|^{\beta} .
$$

Example 4: Consider for all $x \in W$ the homogeneous time-varying system $\left(W \subset B_{1}(0) \subset \mathbf{R}^{n}, W\right.$ is an open and convex set containing the origin)

$$
\dot{x}(t)=-f(t) x(t)\|x(t)\|^{\beta}
$$

with $\beta>0$. Consider the case where the scalar function $f(t)$ is time-periodic with period $T$ and

$$
\bar{f}=\frac{1}{T} \int_{0}^{T} f(t) d t>0 .
$$

Assume there exists $f_{M}>0$ such that for all $t \in \mathbf{R}^{+}$: $\|f(t)\| \leq f_{M}$. Since this is a special case Example 3 $(A(t)=-f(t) I)$, local uniform asymptotic stability of the equilibrium point $x=0$ of (105) follows. This result has also been covered in [8].

\section{Conclusions}

This paper extends results obtained before where local uniform asymptotic stability of an equilibrium of a timevarying differential equation follows from stability properties of its averaged version. It generalises existing averaging results where stability properties had been shown for systems which have a homogeneous vectorfield with order $\tau>0$ [8]. We show that averaging techniques may be useful for systems satisfying appropriate conditions expressed in terms of class $\mathrm{K}$ functions, without requiring a homogeneous or fast time-varying vector field. The results are extensively illustrated by a class of examples.

\section{Acknowledgements}

D.A. has been supported by the Belgian Network DYSCO (Dynamical Systems, Control, and Optimization), funded by the Interuniversity Attraction Poles 
Programme, initiated by the Belgian State, Science Policy Office. The scientific responsibility rests with its authors.

\section{References}

[1] D. AEYELS and J. PEUTEMAN, On exponential stability of nonlinear time-varying differential equations, Automatica, Vol. 35, pp. 1091-1100, 1999.

[2] T. M. APOSTOL, Mathematical Analysis, Addison-Wesley Publishing Company, London, 1974.

[3] J.GUCKENHEIMER, P. HOLMES, Nonlinear Oscillations, Dynamical Systems, and Bifurcations of Vector Fields, Springer Verlag Berlin Heidelberg New York Tokyo, 1983.

[4] L. IANNELLI, K H JOHANSSON, U JÖNSSON, F. VASCA, Averaging of nonsmooth systems using dither, Automatica, Vol. 42,No. 4, pp. 669-676, 2006.

[5] H. K. KHALIL, Nonlinear systems, Prentice Hall, Upper Saddle River, New Jersey, 2002.

[6] R. T. M'CLOSKEY, An averaging theorem for time-periodic degree zero homogeneous differential equations, Systems \& Control Letters, Vol. 32, pp. 179-183, 1997.

[7] R. T. M'CLOSKEY and R. M. MURRAY, Nonholonomic systems and exponential convergence: some analysis tools, Proceedings of the 32th Conference on Decision and Control, San Antonia, Texas, 15-17 december 1993, pp. 943-948.

[8] J. PEUTEMAN and D. AEYELS, Averaging results and the study of uniform asymptotic stability of homogeneous differential equations that are not fast time-varying, Siam Journal on Control and Optimization, Vol. 37, No. 4, pp. 9971010,1999 .

[9] A. R. TEEL, J. PEUTEMAN and D. AEYELS, Semiglobal practical asymptotic stability and averaging, Systems \& Control Letters, Vol. 37, No. 5, pp. 329-334, 1999.

[10] F. VERHULST, Nonlinear Differential Equations and Dynamical Systems 2nd edition, Springer Verlag Berlin Heidelberg New York, 2000. 